\title{
La synonymie dans l'expression de la partition : le trio part, partie et portion
}

\section{Sandrine Stein-Zintz}

\section{Q OpenEdition \\ 1 Journals}

\section{Édition électronique}

URL : https://journals.openedition.org/pratiques/1350

DOI : 10.4000/pratiques.1350

ISSN : 2425-2042

Éditeur

Centre de recherche sur les médiations (CREM)

\section{Édition imprimée}

Date de publication : 15 juin 2009

Pagination : 195-207

\section{Référence électronique}

Sandrine Stein-Zintz, "La synonymie dans l'expression de la partition : le trio part, partie et portion », Pratiques [En ligne], 141-142 | 2009, mis en ligne le 20 juin 2014, consulté le 13 mars 2023. URL http://journals.openedition.org/pratiques/1350; DOI : https://doi.org/10.4000/pratiques.1350 


\title{
La synonymie dans l'expression de la partition : le trio part, partie et portion
}

\author{
Sandrine Stein-Zintz \\ Celted, Université Paul-Verlaine, Metz
}

L'expression de la relation sémantique partie-tout a été très largement envisagée du point de vue des substantifs et nous avons à notre disposition un certain nombre de travaux qui y sont exclusivement consacrés ${ }^{(1)}$. Les trois mots part, partie et portion semblent pourtant sortir du lot, se distinguant tout d'abord par le peu d'intérêt qu'ils suscitent. Même partie, qui fait pourtant figure d'hypéronyme de cette catégorie de substantifs, n'est analysé que dans le cadre de son emploi dans la formule être une partie de, décrite comme la structure d'identification des relations de partie à tout ${ }^{(2)}$. Les analyses sont encore moins nombreuses concernant part $^{(3)}$ et portion ${ }^{(4)}$ qui ne sont que très brièvement cités dans des travaux qui ne leur sont jamais consacrés.

Part, partie et portion ont pourtant un champ d'application plus vaste que celui d'autres partitifs. Ainsi, alors que morceau ou bout ont une distribution qui les lie à des noms concrets, dont les référents sont solides (un morceau/bout de pain), part, partie et portion s'associent à des noms indifféremment abstraits ou concrets et, dans ce cas, dont le référent est solide ou liquide. D'autre part, ces trois mots ont un fonctionnement clairement partitif, à l'inverse d'autres substantifs dont le sémantisme partitif est moins saillant, par exemple endroit qui réfère parfois, mais pas toujours, à la partie d'un tout ( un endroit du mur, par endroits versus Quel endroit magnifique!) ${ }^{(5)}$.

Deux points viennent, par ailleurs, confirmer l'unité de ce trio de partitifs. On

(1) Cf. D. Crévénat (1996) et (1997) sur tranche ou encore C. Venerin-Guenez (2006a) et (2006b) sur les noms de parties issues d'un processus de bris ou de découpe (morceau, bout, fragment).

(2) Concernant la structure être une partie de, nous renvoyons à D. Crevenat \& M. BiermannFischer (1995) et I. Tamba (1994).

(3) Les quelques analyses de part sont par ailleurs nettement déséquilibrées, les dictionnaires et les analyses linguistiques s'attardant très largement sur ses emplois dans des expressions figées, en particulier dans l'organisateur textuel d'une part... d'autre part (cf. B. Combettes (1998), P. Péroz (1998), D. Coltier \& G. Turco (1988)) et l'adverbe de lieu quelque part (cf. C. Blanche-Benvéniste $(2001,2003)$ et G. Kleiber \& F. Gerhard-Krait (2006)), et, inversement, très peu sur le substantif part, sur la base duquel ils sont pourtant construits.

(4) Portion n'a, à notre connaissance, jamais fait l'objet d'un traitement particulier.

(5) Cf. R. Huyghe (2006) à ce propos. 
note tout d'abord une forte circularité dans leurs descriptions lexicographiques, les dictionnaires se renvoyant en boucle les trois mots qui sont présentés comme étant dans une relation synonymique. Ainsi, le Trésor de la Langue Française informatisé (TLFi) décrit part comme une «partie d'un tout considérée sans idée de répartition ou d'attribution particulière » ou bien encore une " portion (d'un tout divisé) attribuée à quelqu'un ou affectée à quelque chose ». A cela viennent s'ajouter des possibilités de substitution qui ne produisent pas, a priori, de changements de sens très importants :

(1) Une part/portion/partie des bénéfices.

(2) Une part/portion/partie du gâteau.

Il suffit pourtant de multiplier les exemples pour rapidement constater que les possibilités de substitution entre les trois mots sont, en réalité, très limitées :

(3) Dans la seconde partie de cet article, nous parlerons de la relation sémantique partie-tout.

*Dans la seconde part de cet article, [...]

* Dans la seconde portion de cet article, $[\ldots]$

(4) JP Morgan Chase a-t-il une part de responsabilité dans la faillite de Lehman Brothers? (Le Monde du 05.10.2008)

*JP Morgan Chase a-t-il une portion de responsabilité dans la faillite de Lehman Brothers?

*JP Morgan Chase a-t-il une partie de responsabilité dans la faillite de Lehman Brothers?

On peut avancer, sans prendre beaucoup de risques, que ces trois mots ne sont pas vraiment synonymes les uns des autres. Il y a pourtant d'importants recoupements, dans leurs emplois respectifs, mais aussi, on peut le supposer, dans leur fonctionnement sémantico-référentiel, sans quoi ils ne permuteraient pas, dans certains exemples, aussi facilement. Nous nous intéresserons donc à part, partie et portion sous une double problématique en essayant de dégager les spécificités de chaque terme, tout en identifiant les points communs qui constituent l'unité de ce trio partitif. Le fonctionnement sémantico-référentiel de chacun de ces mots étant complexe, il serait illusoire de proposer ici une analyse complète de chacun d'eux. Notre objectif sera plus modeste : dégager des pistes de travail qui nous permettront d'esquisser une analyse comparative. Afin d'identifier quelques premiers points communs et différences, nous allons, dans un premier temps, faire un bref bilan des descriptions lexicographiques de nos trois mots.

\section{Les descriptions lexicographiques de part, partie et portion}

L'impression de circularité qui se dégage lors de la consultation des dictionnaires n'est, en réalité, que très limitée. En effet, part, partie et portion connaissent des emplois différents et ne se caractérisent pas par le même nombre de valeurs sémantiques, ce qui implique que les recoupements entre les trois mots ne sont que partiels. Voyons cela en détail.

\subsection{Part}

Les différents dictionnaires que nous avons consultés ${ }^{(6)}$ organisent leur description d'une façon identique, en proposant une bipartition des emplois de part: les emplois spatiaux de ce mot sont opposés à des emplois non spatiaux.

(6) Le Grand Larousse de la langue française ( GLLF), le Trésor de la Langue Française infor- 
Sous les emplois spatiaux, qui découlent d'une origine sémantique spatiale ${ }^{(7)}$, nous retrouvons un panel varié d'expressions figées, en particulier le paradigme adverbial autre part/quelque part/nulle part, les locutions adverbiales et prépositionnelles de part en part, de part et d'autre de, de toute(s) part(s) ou bien encore l'organisateur textuel d'une part... d'autre part. Il est à noter que le sens spatial de part n'a survécu que par le biais de ces locutions figées.

Les emplois non spatiaux de part regroupent un emploi nominal, très souvent illustré par le biais du syntagme nominal une part de tarte, ou encore une part de responsabilité, ainsi qu'un certain nombre de formes figées, par exemple prendre part, faire part, pour une part...

Cette bipartition des emplois de part doit pourtant être manipulée avec précaution et ceci au moins pour deux raisons :

i) l'opposition spatial/non spatial n'a pas toujours été celle que nous connaissons aujourd'hui. La prise en compte de l'aspect diachronique indique en effet que cette opposition s'affaiblit, voire disparaît totalement si l'on prend en compte des états antérieurs de la langue. Ainsi, la locution d'une part... d'autre part, qui ne connaissait qu'un emploi spatial en ancien français, n'est plus employé aujourd'hui que dans une fonction d'organisateur textuel ${ }^{(8)}$.

ii) les emplois spatiaux s'analysent, au même titre que les emplois non spatiaux, sous l'angle de la partition. Comme nous l'avons montré ailleurs ${ }^{(9)}$, il n'y a pas d'un côté les emplois partitifs de part et de l'autre côté des emplois non partitifs.

Concernant notre propos, à savoir la relation entre part, partie et portion, nous constatons que ce ne sont que les emplois non spatiaux de part qui entretiennent une relation synonymique avec partie et portion, l'emploi spatial de part entrant en concurrence, non pas avec ces deux partitifs, mais avec côté, par le biais, par exemple, des locutions d'une part... d'autre part (opposé à d'un côté... d'un autre côté) et de toute(s) part(s) (opposé à de tout(s) côté(s)).

Voyons à présent ce que les dictionnaires nous disent de partie.

\subsection{Partie}

Les dictionnaires distinguent trois valeurs pour partie, chacune d'entre elles s'accompagnant d'un nombre important de locutions figées ${ }^{(10)}$.

matisé (TLFi) ainsi que le Dictionnaire alphabétique et analogique de la langue française de P. Robert.

(7) Polysémique en ancien français, part pouvait être employé dans le sens contemporain de partie (une part du temple) mais également sous une acception spatiale dans le sens de côté:

An cele lande avoit un gué et d'autre part armez estoit uns chevalier qui le gardoit (Le chevalier de la Charrette, Chrétien de Troyes, 1177).

Dans cette lande il y avait un gué et de l'autre côté se tenait armé un chevalier qui montait la garde (traduction de C. Méla).

En ancien français, le mot costé n'est employé que pour désigner la côte, partie de la cage thoracique. Ce n'est qu'à partir de la période de moyen français que costé est employé pour référer plus largement aux côtés d'un objet.

(8) Cf. B. Combettes (1988) à ce propos.

(9) Cf. S. Stein-Zintz (2008).

(10) Faute de place, nous ne les mentionnons pas en détail ici. En voici néanmoins un petit échantillon: faire partie de, en partie, être partie prenante, avoir affaire à forte partie, abandonner la partie... 
La première valeur est clairement partitive. Le $T L F i$ et le GLLF proposent respectivement les gloses «élément constitutif ou portion d'un tout » (TLFi), "portion d'un ensemble concret ou abstrait considéré comme élément concourant à former un tout » $(G L L F)$ :

(5) Une partie des récoltes (TLFi).

La seconde valeur n'est plus présentée sous l'angle de la partition. Les dictionnaires proposent les gloses «personne qui plaide » $(G L L F)$ ou plus largement «personne qui participe » $(T L F i)$ :

(6) Le mariage est un contrat et il se dissout par le consentement des parties (TLFi).

La troisième valeur, qui n'est pas plus que la seconde présentée sous l'angle de la partition, réfère à un « projet » $(G L L F)$, à une « activité », un « divertissement » (TLFi) :

(7) Ils entrèrent gaiement dans la petite ville dont les platanes annonçaient déjà la chaleur et les parties de boules du Midi (TLFi).

Les deux valeurs « personne qui participe » et « divertissement» ne sont pas représentées par part et portion. Ainsi, seule la première valeur de partie ouvre des possibilités de substitution avec part ou portion:

(8) une partie/portion/part de la récolte

(9) * Le mariage est un contrat et il se dissout par le consentement des parts/portions.

(10) *les parts/portions de boules du Midi

Deux éléments nous indiquent pourtant que les recoupements entre part, portion et partie méritent un examen plus approfondi.

Il faut tout d'abord veiller à ne pas exclure trop rapidement les emplois de partie qui ne sont pas présentés sous l'angle de la partition, en particulier la seconde valeur " personne qui participe ». On note ainsi d'intéressantes connexions entre la locution être partie prenante (associée par les dictionnaires à la valeur «personne qui participe ») et certains emplois de part, en particulier la forme figée prendre part, synonyme de participer.

On constate par ailleurs que les possibilités de substitution sont limitées, y compris dans des emplois clairement partitifs, ce qui laisse supposer un fonctionnement partitif différent :

(11) les différentes parties d'un château-fort (TLFi)

*les différentes portions d'un château-fort

*les différentes parts d'un château-fort

Nous reviendrons sur ce point plus en détail, dans la seconde partie du travail.

\subsection{Portion}

Les dictionnaires distinguent différents emplois qui sont tous présentés sous l'angle de la partition. Ainsi, d'après le $T L F i$, une portion est « une part, une quantité d'un mets destiné à une personne (une portion de choucroute) », " un élément, une partie d'un ensemble généralement homogène (une portion de route)» ou bien encore « une partie d'argent, de biens qui revient à quelqu'un (une portion d'héritage) ». Par ailleurs, portionn'est à l'origine que de deux formes figées (une demiportion, portion congrue), ce qui contraste avec l'abondance de formes figées construites avec part et partie. 


\subsection{Bilan}

En résumé, nous retiendrons que les recoupements sémantiques entre part,partie et portion sont limités :

i) part se distingue, en étant le seul à connaître des emplois spatiaux. Etant de surcroît figés, ils excluent toute permutation avec portion et partie. Il conviendra par contre d'examiner l'ampleur des recoupements avec partie et portion à partir des emplois non spatiaux du type une part de tarte, une part d'héritage. ii) d'après les dictionnaires, partie est le membre de notre trio dont le sémantisme est le plus varié. Les différents emplois de partie sont diversement touchés par la concurrence avec portion et part. Part vient concurrencer partie dans son acception «élément constitutif d'un tout» mais également " personne qui participe » alors que portion ne vient concurrencer partie que dans son acception «élément d'un tout» ${ }^{(11)}$. Quant à la valeur activité (partie de football), elle semble être spécifique à partie.

iii) portion est, a priori, le seul à ne pas connaître d'emploi qui lui soit vraiment spécifique.

Ce premier bilan indique que si, d'un côté, chaque terme a un champ d'application différent, ce sont, d'un autre côté, les aspects partitifs qui constituent l'unité de cette famille de mots. Certaines questions restent néanmoins en suspens :

i) si part, partie et portion présentent un sémantisme partitif, les dictionnaires restent très vagues quant aux «touts » auxquels ils sont associés. Prenons l'exemple de part: une part est sommairement définie comme une «partie d'un tout » (TLFi), « une partie en général » (Hatzfeld et Darmesteter) ou encore « une partie, portion d'un tout quelconque » (Littré),... Mais, quel est ce tout? Est-il le même pour portion et partie?

ii) la notion de partition reste très vague dans les descriptions lexicographiques de part, partie ou portion. Or, il existe différentes modalités de partition. Par exemple, C. Venerin-Guenez (2006a) distingue les «noms de parties brisées » (op. cit. : 9), qui résultent de la " division ou séparation non naturelle d'un tout originel, par destruction du référent du tout» (op. cit. : 9), des «noms de parties découpées $[\ldots]$ qui servent de désignation pour des entités résultant de processus de séparation volontaire » (op. cit. : 9). Dans ce cas, quelles sont les spécificités des opérations de partitions auxquelles participent part, partie et portion?

Nous commencerons par nous intéresser au premier de ces deux points : 1'examen des touts auxquels ces trois mots peuvent ou, le cas échéant, ne peuvent pas s'associer, va nous permettre de poser les bases de notre analyse comparative.

\section{Examen des touts associés à part, partie et portion}

Part, partie et portion appartiennent à la catégorie des substantifs syncatégorématiques ce qui signifie qu'ils sont non-autonomes référentiellement : ils dépendent d'un autre élément pour se fixer référentiellement. Dans de très nombreux exemples, l'expression nominale de cet élément figure à leur droite, dans une structure syntaxique de la forme dét part/partie/portion de (dét) $\underline{N}^{(12)}$ :

(11) Nous n'avons relevé aucun emploi de portion dans le sens de «personne qui participe ».

(12) N renvoie au nom du tout soumis à partition. 
(12) Le pare-choc est une partie de la voiture.

(13) J'aimerais manger une part de tarte.

(14) Le plus important séisme qui ait frappé l'Algérie depuis deux décennies a fait trembler la terre sur une large portion de littoral, mercredi soir 21 mai, touchant Alger et plusieurs villes à l'Est de la capitale (Le Monde du 23.05.2003).

Dans ces exemples, la relation entre voiture, tarte, littoral d'un côté et partie, part, portion de l'autre est une relation de tout à partie.

On constate très nettement que c'est partie qui est touché par le moins de contraintes concernant N. Partie s'emploie avec une variété intéressante de noms de touts dont les référents sont indifféremment spatiaux (une partie de la région), temporels (une partie de la journée), massifs (une partie de la farine), comptables (une partie de la voiture) ou bien encore collectifs (une partie de la foule) ${ }^{(13)}$. Partie témoigne d'une grande souplesse d'emploi. Mais ce partitif n'en est pas pour autant un «joker épilinguistique » ${ }^{(14)}$. I. Tamba (1994) précise ainsi que partie est incompatible avec les $\mathrm{N}$ à référent animé : *le dos est une partie de l'homme, *le groin est une partie du sanglier. D'après l'auteur, seule l'introduction d'un relais nominal inanimé (par exemple dos ou bien encore tête) permet de lever cette restriction d'emploi : le dos est une partie du corps humain, le groin est une partie de la tête du sanglier.

L'examen de notre corpus n'a révélé aucune contrainte supplémentaire. Partie semble s'employer avec presque tout type de N, la seule contrainte que nous relevons concernant, non pas N, mais sa détermination. En effet, partie exige la présence d'un déterminant avant les $\mathrm{N}$ à référence massive et les $\mathrm{N}$ pluriels, ce qui n'est, par contre, pas le cas de part et de portion:

(15) une partie du riz versus *une partie de riz (une part/portion de riz)

(16) une partie des fraises versus *une partie de fraises (une part/portion de fraises)

A l'inverse de partie, part et portion ne s'emploient pas avec tout type de N. Certaines contraintes sont d'ailleurs communes aux deux mots. Ainsi, l'emploi de part et portion pose problème avec de nombreux $\mathrm{N}$ comptables :

(17) Dans la majorité des accidents graves ou mortels, les traumatismes sont dus au fait que les charges ou les accélérations subies par une partie de la voiture sont supérieures à ce que peut tolérer le corps (Rapport mondial sur la prévention des traumatismes dus aux accidents de la circulation, 2004).

* les accélérations subies par une portion de la voiture

* les accélérations subies par une part de la voiture

Part et portion semblent mieux s'adapter aux N massifs :

(18) une portion de pain d'épices

(19) Mettre dans un shaker deux parts de jus d'ananas, une part de lait de coco, une part de rhum, un peu de sirop de sucre et une lampée de Curaçao pour la couleur.

La proximité entre part et portion est néanmoins limitée. Certaines contraintes ne concernent que part, d'autres sont propres à portion. En voici un petit échantillon :

—à l'inverse de portion, mais aussi de partie, part s'emploie très difficilement aux côtés de N spatiaux :

(13) Cette typologie, non exhaustive, est ici volontairement éclectique afin d'insister sur la grande diversité des touts auxquels partie peut s'associer.

(14) Nous empruntons cette expression à I. Tamba (1994). 
(20) Vers $22 \mathrm{H} 00$, onze routes départementales de l'Isère étaient coupées à la circulation, ainsi qu'une portion de la RN 532 entre Beauvoir et Izeron, soit en raison de coulées de boue, soit « à titre préventif », selon la même source (Le Monde du 07.06.2002).

Onze routes départementales de l'Isère étaient coupées à la circulation, ainsi qu'une partie de la RN 532 entre Beauvoir et Izeron

* Onze routes départementales de l'Isère étaient coupées à la circulation, ainsi qu'une part de la RN 532 entre Beauvoir et Izeron.

(21) Ce nouvel élément de l'enquête semble innocenter encore un peu plus les pilotes russes, montrés du doigt quelques heures après la catastrophe notamment par un ministre régional allemand et les aiguilleurs du ciel suisses, chargés de contrôler cette portion de l'espace aérien, pourtant en territoire allemand (Le Monde du 09.07.2002).

Les aiguilleurs du ciel suisses [...] chargés de contrôler cette partie de l'espace aérien.

*Les aiguilleurs du ciel suisses [...] chargés de contrôler cette part de l'espace aérien.

— d'autres contraintes touchent portion mais épargnent part. Par exemple, part s'emploie très fréquemment dans une structure syntaxique du type la/une part de N1 dans N2, configuration interdite à portion :

(22) La part de lait dans ce chocolat est de $10 \%$.

* La portion de lait dans ce chocolat est de $10 \%$.

(23) On m'attribue une part de responsabilité dans la situation actuelle de la Corse (L'Express du 12.02.1998).

*On m'attribue une portion de responsabilité dans la situation actuelle de la Corse.

En résumé, nous retiendrons que part, partie et portion ne s'emploient pas avec les mêmes touts. Il faudrait bien entendu procéder à une analyse plus approfondie, ce que nous ne pouvons faire en détail ici, mais, d'ores et déjà, plusieurs pistes se dégagent :

i) alors que partie s'emploie avec un éventail varié de touts, l'emploi de portion et de part est plus contraint.

ii) l'inadéquation de part et de portion avec certains touts, par exemple certains $\mathrm{N}$ comptables, nous pousse à nous interroger sur les spécificités partitives des touts en question. Ainsi, on peut confronter l'opposition massif/comptable à la relation sémantique partie/tout : les référents des $\mathrm{N}$ massifs ont une structuration interne différente des référents des $\mathrm{N}$ comptables ${ }^{(15)}$. On peut donc légitimement penser que les partitions appliquées à ces deux types de touts présentent des différences.

Mais, si l'examen du N associé à part, partie et portion pose les premiers jalons de notre analyse comparative, il pose également d'emblée deux problèmes.

La première difficulté concerne la caractérisation des $\mathrm{N}$ exclus ou admis après part, partie et portion car les contraintes que nous relevons ne correspondent pas à

(15) Le référent d'un nom comptable présente des limites externes ainsi qu'une structuration interne hétérogène alors que le référent d'un nom massif ne présente ni limites externes, ni limites internes puisque sa structuration interne est homogène. L'opposition homogène/hétérogène est identifiée par le test de divisibilité : « est massif le nom qui dénote une entité dont toute partie donne une entité de même type : si j'enlève de l'eau à de l'eau, c'est encore de l'eau, un morceau de sucre, c'est encore du sucre » (G. Kleiber, 1997 : 322). Par contre, la roue d'une voiture, ce n'est plus une voiture : la structuration interne du référent de voiture est hétérogène. 
certaines classifications traditionnelles des substantifs, par exemple l'opposition massif/comptable ou l'opposition abstrait/concret. Prenons l'exemple de part : une même catégorie se trouve à la fois exclue et en même temps admise dans les SN part de $N$. Ainsi, si part s'emploie mal avec certains N comptables (*une part de voiture), d'autres comptables ne posent aucun problème (une part de tarte). L'opposition concret/abstrait n'est pas plus opérante : parts'associe avec certains $\mathrm{N}$ abstraits (une part de responsabilité) mais pas avec d'autres (*une part de guérison).

Nous constatons par ailleurs qu'un même N, est, en fonction du contexte, exclu ou admis dans les syntagmes part/partie/portion de $N$, ce qui rend quasi impossible leur distinction sur la base du $\mathrm{N}$ auquel ils sont associés :

(24) *Dans la majorité des accidents graves ou mortels, les traumatismes sont dus au fait que les charges ou les accélérations subies par une part de la voiture sont supérieures à ce que peut tolérer le corps.

(25) La part de la voiture dans les déplacements quotidiens augmente, tandis que les modes non motorisés connaissent une baisse régulière (Rapport de l'Agence de l'Environnement et de la Maîtrise de l'Energie, août 2000).

(26) *Une part du coton produit ces dernières années est génétiquement modifié.

(27) Il y a une part de coton dans ce tissu.

L'examen du N après part, partie et portion n'est donc pas suffisant pour décrire le fonctionnement de chacun de ces mots. Dans la section suivante, nous allons voir que pour caractériser le fonctionnement sémantico-référentiel de part, partie et portion, il est tout aussi important de s'intéresser aux modalités des partitions auxquelles participent nos trois termes.

\section{Des modalités de partitions différentes}

L'objectif de cette partie est de mettre en relief les spécificités des opérations de partition dont sont issues des parts, des parties ou des portions.

Nous montrerons successivement dans cette section que, à l'inverse de partie, les partitions auxquelles participent part, mais aussi portion, sont :

$1^{\circ}$ des partitions arbitraires, i.e. ne respectant pas les limites internes de l'objet soumis à partition.

$2^{\circ}$ des partitions dont le résultat est destiné à un ou plusieurs individus.

\subsection{Part et portion : des partitions arbitraires}

Partons des substantifs tarte et voiture. Les référents de ces deux substantifs se caractérisent par une structuration interne hétérogène ${ }^{(16)}$, se composant chacun de plusieurs parties différentes : de la croûte, de garniture, de la meringue,... pour le référent de tarte et un pare-choc, des roues, une calandre, ... pour le référent de voiture. Il est possible d'appliquer à ces deux objets deux types de partition :

- une partition respectant leurs limites internes :

(28) Si je fais une tarte au citron, je ne fais cuire qu'une partie de la tarte, c'est-à-dire la croûte. Ce n'est qu'après que je rajoute la garniture.

(29) Le pare-choc est une partie de la voiture.

(16) Nous reprenons ici la terminologie proposée par G. Kleiber (1997). 
- une partition ne respectant pas leurs limites internes :

(30) Je découpe la tarte au citron en quatre parts.

(31) L'accident a été si violent que la voiture s'est disloquée en deux parties.

Ni part, ni portion ne pourront être employés dans des partitions qui impliquent un découpage du premier type :

(32) Si je fais une tarte au citron, je ne fais cuire qu'une *part/*portion de la tarte c'està-dire la croûte. Ce n'est qu'après que je rajoute la garniture.

(33) Le pare-choc est une *part/*portion de la voiture.

Notre hypothèse se vérifie également dans les opérations de composition d'un tout qui appartiennent, tout autant que les opérations de décomposition (i.e. les découpages), à la sphère de la relation sémantique partie-tout. Part et portion s'emploient ainsi avec des verbes qui impliquent la perte d'individualité des éléments participant à la composition (le tout construit présente une structure interne homogène), comme mêler ou mélanger:

(34) Pour faire du caramel, il faut mélanger deux parts de sucre et une part d'eau.

Partie, quant à lui, ne peut être employé qu'avec des verbes comme assembler, relier qui impliquent que les parties gardent leur individualité propre à l'intérieur du tout construit, ce dernier présentant une structuration interne hétérogène :

(35) Puisque nous parlons de ces vis, disons aussi deux mots de celles dont on se sert pour assembler les diverses parties du billard (Nosban 1857 dans Frantext).

(36) *Puisque nous parlons de ces vis, disons aussi deux mots de celles dont on se sert pour assembler les diverses parts du billard.

Par ailleurs, on note que les parts ou portions issues d'une même partition sont qualitativement identiques. Reprenons l'exemple avec tarte. Lorsque l'on découpe une tarte en parts ou en portions, on ne suit pas les limites internes du référent de tarte. Ainsi, si je découpe une tarte aux fraises pour mes convives, je ne donne pas à l'un la croûte et à l'autre la garniture aux fraises. Chaque part/portion issue du découpage d'une tarte présente les mêmes caractéristiques : de la croûte avec de la garniture aux fraises. Si mon découpage peut avoir comme résultat des parts/portions inégales (si je découpe mal, j'obtiens des parts/portions plus grandes que d'autres), elles seront néanmoins toutes qualitativement identiques.

Cette seconde caractéristique explique d'ailleurs certaines des contraintes d'emplois qui touchent part et portion, par exemple avec le $\mathrm{N}$ voiture. Ces deux mots ne peuvent participer qu'à des partitions produisant des parts, des portions identiques. Or, la partition du référent de voiture ne peut avoir comme résultat que des parties qualitativement différentes. Ainsi, si dans une partie arbitraire de la voiture (je coupe ma voiture en deux) on trouve le moteur, les autres parties en seront dépourvues. C'est, d'après nous, ce qui explique l'incompatibilité de part et de portion avec un tout comme le référent de voiture.

Il est pourtant possible de contourner la contrainte qui pèse sur part avec le $\mathrm{N}$ voiture:

(37) En cas de séparation, Marie pourra demander sa part de la voiture.

Mais, la partition opérée dans cet exemple est différente de celle opérée par partie dans les exemples (29) ou (31) : le référent de voiture n'est plus matériellement 
découpé, la partition ne concernant plus l'objet mais sa valeur. Partie, tout comme portion, est d'ailleurs tout à fait exclu dans ce type de contexte :

(38) *En cas de séparation, Marie pourra demander sa partie de la voiture.

(39) *En cas de séparation, Marie pourra demander sa portion de la voiture.

L'exemple (37) met en lumière une autre spécificité de part : l'emploi du possessif nous oriente vers des questions concernant la finalité de l'opération de partition en jeu.

\subsection{Part et portion : des partitions qui impliquent un destinataire}

Part et portion s'emploient avec des prédicats partitifs qui supposent un agent, un sujet animé humain comme diviser, partager, couper ou encore découper :

(40) J'ai découpé la tarte en six parts.

(41) J'ai découpé la tarte en six portions.

C. Venerin-Guenez (2006a) parle à ce propos de « séparation volontaire» (op. cit. : 9).

Il est par contre exclu pour part, tout comme portion d'ailleurs, d'être employé dans le cadre d'une partition qui n'implique aucun agent à la source du découpage :

(42) *La tarte est tombée et s'est brisée en six parts.

(43) * La tarte est tombée et s'est brisée en six portions.

Partie, quant à lui, ne semble pas être touché par cette contrainte :

(44) Le pétrolier s'est d'abord brisé en deux parties mardi, avant de couler complètement vers 16h30 locales (dépêche AFP du 19.11.2002).

Pourtant, je peux délibérément détruire la tarte que je viens de confectionner. Il y a donc un agent à la source de la partition. Mais s'il en résulte des morceaux, des bouts, des parties, ces dernières ne pourront en aucun cas être qualifiées de parts ou de portions : je ne peux parler de parts ou de portions que si je les destine à quelqu'un.

Les dictionnaires nous mettent sur la piste d'un lien sémantique fort entre part, portion et la notion de distribution, d'attribution ${ }^{(17)}$. Une part est « une portion (d'un tout divisé) attribuée à quelqu'un ou affectée à quelque chose » (TLFi), «ce qui revient à quelqu'un » (Le Robert). La notion d' « attribution » apparaît comme le fil conducteur sur lequel est bâti une grande partie de la description lexicographique de part.

Le cotexte de part est d'ailleurs riche en marques linguistiques qui se rattachent directement au champ sémantique de «l'attribution ». On trouve ainsi très souvent aux côtés de part :

- les verbes attribuer ou donner :

(45) S'attribuer une part du gâteau.

(46) On m'attribue une part de responsabilité dans la situation actuelle de la Corse (L'Express du 12.02.1998).

(47) La formation donne une part importante à la pratique en entreprise : les élèves effectuent 14 semaines de stage de mai à septembre entre la première et la deuxième année (Document de l'académie de Nancy-Metz).

(17) Il est à noter que la notion d' « attribution » entretient des liens sémantiques avec l'opération de partition : le verbe attribuer est défini par le TLFi comme «donner à quelqu'un une chose dans un partage, une répartition, une adjudication, comme sa part, son lot, etc. ». 
- une détermination possessive. De l'attribution d'une part découle une relation de possession entre cette part et son destinataire ${ }^{(18)}$ :

(48) Ne touche pas à ma part de tarte.

(49) Chacun sur terre a sa part de peine.

- un complément du nom qui réfère à un possesseur dans une structure de la forme la part de + nom propre ${ }^{(19)}$ :

(50) Ne touche pas à ça, c'est la part de Paul.

Portion, tout comme part, doit s'envisager sous ces aspects : une portion est en effet définie comme une «quantité, une part de nourriture destinée à une personne », une « part qui revient à quelqu'un ». C'est d'ailleurs l'hypothèse que défendent D. Crévenat \& M. Biermann-Fisher (1995) :

Les mots portion et ration expriment la partition mais ils l'orientent en ce sens qu'ils impliquent un destinataire [...]. Beaucoup de produits alimentaires sont conditionnés en portions pour une, deux, trois personnes : on connaît les portions de Vache qui rit, petits triangles ou carrés préemballés de fromage fondu. [...] Le terme portion dénomme donc une entité calculée et destinée à un ou plusieurs individus. (M. Biermann-Fischer \& D. Crévenat-Werner, 1995).

On parle ainsi de portions individuelles, de portions pour X personnes :

(51) La brioche $\mathrm{X}$ est un produit en portions individuelles, prêt à consommer : il se réchauffe quelques secondes au four micro-ondes ou quelques minutes au four traditionnel.

(52) Pour griller une carpe avec réussite, il est nécessaire qu'elle pèse au moins un kilo, ce qui correspond à une portion pour quatre personnes.

Mais, si la notion d' "attribution » constitue un point commun important entre part et portion, c'est également sur ce terrain que ces deux partitifs se distinguent.

Tout d'abord, il faut noter que la notion d' « attribution » n'est pas présente dans tous les emplois de portion. C'est le cas de ses nombreux emplois spatiaux qui excluent d'ailleurs toute substitution avec part (Cette portion d'autoroute est accidentogène/*Cette part d'autoroute est accidentogène). Dans cet exemple, portion se rapproche plus de partie que de part (Cette partie d'autoroute est accidentogène).

La notion d' " attribution » implique qu'un troisième élément s'invite dans la description sémantique de ces deux mots, en plus des deux notions de tout et de partie généralement convoquées. Nous avons vu supra que ce troisième élément peut être envisagé comme un destinataire (mapart de tarte, une portion pour trois personnes). Mais, dans de nombreux exemples, il est difficile de parler de destinataire car on ne reçoit plus rien et, au contraire, on donne quelque chose :

(18) Certaines locutions figées sont d'ailleurs obligatoirement composées d'un possessif (pour ma part, de sa part).

(19) Le destinataire d'une part sous la forme d'un complément du nom figure d'ailleurs dans les deux locutions suivantes :

- la part du Lion: d'après le TLFi, il s'agit de « la plus grosse part que s'adjuge abusivement le plus fort, le plus autoritaire ", par allusion à une des fables de La Fontaine, La Génisse, la Chèvre et la Brebis en société avec le Lion.

- la part du pauvre, "part de repas, notamment de galette des rois, qui était mise de côté pour l'arrivée éventuelle d'un pauvre» $(T L F i)$. Cette locution connaît un synonyme vieilli, la part de la Vierge. 
(53) Le colocataire qui a signé le bail a, entre autres, l'obligation de payer sa part du loyer.

(54) fournir sa part d'efforts

Or, ce type de contexte exclut l'emploi de portion:

(55) *Le colocataire qui a signé le bail a, entre autres, l'obligation de payer sa portion du loyer.

(56) *fournir sa portion d'efforts

Cette différence dans l'emploi de part et de portion indique non seulement une nuance de sens entre les deux mots, qu'il conviendrait bien entendu de décrire plus précisément, mais indique également que la notion d' "attribution » demande, elle aussi, un complément d'analyse. Cette piste de travail montre surtout que les substantifs partitifs, part et portion dans notre cas, gagneraient à être analysés au-delà des strictes notions de partie et de tout.

\section{Bilan}

Toute 1'originalité de ce trio partitif est qu'un même point commun concerne très rarement les trois mots à la fois mais rapproche très souvent deux lexèmes, isolant le troisième. Ainsi, tour à tour, les points communs rapprochent tantôt part de portion ou bien encore portion de partie, en excluant part. Cette étude mériterait de nombreux approfondissements mais plusieurs grandes lignes se dégagent:

i) c'est entre partie et part que l'on relève le plus de différences. Ces deux mots sont finalement très peu en concurrence alors même qu'ils ont des origines morpho-sémantiques communes.

ii) c'est entre part et portion que la proximité sémantique est la plus forte. Ces deux mots ont en commun certaines contraintes d'emplois qui s'expliquent par un fonctionnement sémantico-référentiel proche, le point commun le plus important que nous ayons relevé concernant l'affranchissement des limites internes des touts soumis à partition. Mais portion n'en est pas pour autant un doublon de part. Si, d'un côté, certains points laissent entrevoir de fortes relations avec part (la finalité de la partition au travers d'une distribution), d'un autre côté, portion s'en éloigne très nettement, en présentant plus de similitudes avec partie, dans les emplois spatiaux par exemple.

Plus largement, ce travail aura également montré que l'examen des substantifs partitifs doit se faire au-delà de leur stricte relation avec les touts auxquels ils sont associés. Il faut tout autant s'intéresser aux modalités des opérations de partition ainsi qu'à leur finalité. 


\section{Références bibliographiques}

BLANCHE-BENVENISTE, C. (2001) : «Grammaticalisation d'un terme de lieu :quelque part et mis à part», Recherches sur le français parlé, 16, 83-101.

- 2003, "Quelqu'un, quelque chose, quelque part, quelquefois », Verbum, 25, 3, pp. 277-290.

CombetTes, B. (1998) : "Approche diachronique des tours corrélatifs du type : d'une part... d'autre part», in C. Schnedecker (éd.), Les corrélats anaphoriques, Paris, Klincksieck, pp. 37-58.

CREVEnAT-WERnER, D. (1996) : «Tranche avec les noms concrets », Cahiers de lexicologie, 68, pp. 45-61.

- (1997, « Comment peut-on avoir une tranche abstraite ? "Verbum, 19, 3, pp. 275-292.

Crevenat-Werner, D., \& Biermann-Fischer, M. (1995) : « Les noms massifs concrets et la structure "être une partie de" ", Arba, 5, pp. 107-124.

FRANTEXT - Nancy : A TILF [en ligne] http ://www.frantext.fr

Grand Larousse de la langue française en sept volumes, 1989, Paris, Larousse.

HATZFeld, A. \& DARMesteter, A. (1890) : Dictionnaire général de la langue française du commencement du XVII jusqu'à nos jours, Paris, Delagrave.

HuYGHE, R. (2006) : «Entre localisation et partition : le cas de endroit », in G. Kleiber, C. Schnedecker, A. Theissen (éds), La relation partie-tout, Louvain, Peeters, pp. 41-58.

KleIBER, G. (1997) : «Massif/comptable et partie/tout », Verbum, 19, 3, pp. 321337.

KLEIBER, G., \& GERHARD-KRAIT, F. (2006) : «Les emplois émergents non spatiaux de quelque part », in G. Kleiber, C. Schnedecker, A. Theissen (éds), La relation partie-tout, Louvain, Peeters, pp. 89-106.

LiTTRÉ, E. (1872) : Dictionnaire de la langue française, CD-Rom Texte Intégral, Marsanne, Redon.

PÉROZ, P. (1998) : «D'une part, d'autre part : le partage du dit », in C. Schnedecker (éd.), Les corrélats anaphoriques, Paris, Klincksieck, pp. 61-73.

ROBERT, P. (1981) : Dictionnaire alphabétique et analogique de la langue française, Paris, Le Robert (Edition corrigée).

STEIN-ZINTZ, S. (2008) : Part: de la méronymie à la segmentation discursive. Analyse sémantico-discursive des emplois nominaux et adverbiaux d'une expression partitive atypique en français contemporain, Thèse de doctorat nouveau régime, Université de Metz.

TAMBA, I. (1994) : «Un puzzle sémantique : le couplage des relations de tout à partie et de partie à tout », Le gré des langues, 7, pp. 64-85.

TLFI - Trésor de la langue française informatisé. Nancy : ATILF [en ligne] http ://atilf.atilf.fr/tlf.htm

Turco, G. \& COLTIER, D. (1988) : «Des agents doubles de l'organisation textuelle, les marqueurs d'intégration linéaire », Pratiques, 57, pp. 57-79.

VENERIN-GUENEZ, C. (2006a) : Morceau, bout, fragment, tranche, etc. Les noms des parties atypiques issues d'un processus de bris ou de découpe, Thèse de doctorat, Université de Lille 3.

- (2006b) : «Noms de parties séparées et prédicats de destruction », in G. Kleiber, C. Schnedecker, A. Theissen (éds), La relation partie-tout, Louvain, Peeters, pp. 381-398. 